\title{
The emergency surgical infection management checklist of hospitalized patients with coronavirus disease 2019 (COVID-19) in Zhejiang, China
}

\author{
Lingmei $\mathrm{Ni}^{1}$, Yanan Zhou $^{1}$, Zuowei $\mathrm{Ni}^{1}$, Yifang $\mathrm{Ma}^{2}$, Haiting Fen ${ }^{1}$, Fang Wang ${ }^{1}$, Sheng $\mathrm{Zhang}^{1}$, \\ Xiaofang Fei ${ }^{1}$, Qunying Pan ${ }^{1}$, Xinzheng Hong ${ }^{1}$, Tingting $\mathbf{Q u}^{1}$, Zifeng Zhong ${ }^{1}$, Zuobing Chen ${ }^{3}$ \\ ${ }^{1}$ Department of Infection Prevention and Control, First Affiliated Hospital, Zhejiang University School of Medicine, Hangzhou, China; ${ }^{2}$ Department \\ of Operating Room, First Affiliated Hospital, Zhejiang University School of Medicine, Hangzhou, China; ${ }^{3}$ Department of Rehabilitation Medicine, \\ First Affiliated Hospital, Zhejiang University School of Medicine, Hangzhou, China \\ Correspondence to: Zuobing Chen. Department of Rehabilitation Medicine, First Affiliated Hospital, Zhejiang University School of Medicine, 79 \\ Qingchun Road, Hangzhou 310003, China. Email: czb1971@zju.edu.cn.
}

\begin{abstract}
The novel coronavirus disease 2019 (COVID-19) epidemic broke out in 2019, it is highly contagious, and the infection rate among medical staff is high. The management of infection prevention and control during emergency surgery of COVID-19 patients has been outlined and the perioperative infection management checklist for emergency surgery of COVID-19 patients has been summarized and validated. There have been 13 emergency surgeries performed on COVID-19 patients at our hospital during this time. Two cases were cured and discharged, and the others were discharged after improvement and transferred to further rehabilitation, 30-day mortality of the emergency surgical is $0 \%$. Once the emergency surgery protocol in the hospital is successfully established, emergency surgery can be performed as soon as the surgical planning decision is made, and the operating room can be prepared for use at any time. The incidence of surgical site infection (SSI) was largely higher than that of ordinary patients in the same time; however, the successful implementation of emergency surgery for COVID-19 had positive significance in reducing the incidence of death, risk of bleeding, and hypoxia. The current cumulative cure rate of COVID-19 in our hospital is $98 \%$, patient mortality rate is $0 \%$, and the incidence of COVID-19 infection in medical staff is $0 \%$. The emergency surgical infection management checklist is feasible and effective in guiding the preoperative and intraoperative surgical procedures.
\end{abstract}

Keywords: Coronavirus disease 2019 (COVID-19); infection management; emergency surgery; checklist

Submitted Jan 14, 2021. Accepted for publication Mar 23, 2021.

doi: $10.21037 /$ apm-21-430

View this article at: http://dx.doi.org/10.21037/apm-21-430

\section{Introduction}

Thresholds for surgery during the SARS-CoV-2 pandemic should be higher than during normal practice, Mortality and pulmonary complications in patients undergoing surgery with perioperative SARS-CoV-2 infection study show the 30 -day mortality of SARS-CoV-2 infection patients were $23.8 \%$ (1). To date, the number of patients infected with highly contagious coronavirus disease 2019 (COVID-19) continues to increase rapidly. It has been identified as a Class B infectious disease and further managed as a Class
A infectious disease by the Chinese government (2). The coronavirus can be transmitted via direct contact and person-to-person transmission of respiratory droplets (3), and it has a mean incubation period of 6.4 days (4). It poses a great concern to global health (5). At present, the mortality rate of COVID-19 in China is $2.3 \%$, which is much less than the $9.6 \%$ for severe acute respiratory syndrome (SARS) or the $34.4 \%$ for Middle East respiratory syndrome (MERS) (6), but higher than that of seasonal flu, which is less than $1 \%$. 
Table 1 The incision infection rate in 13 surgery patients with coronavirus disease 2019 (COVID-19) at the First Affiliated Hospital, Zhejiang University

\begin{tabular}{|c|c|c|c|}
\hline & All patients & $\begin{array}{l}\text { Incision } \\
\text { infections }\end{array}$ & $P$ value \\
\hline \multicolumn{4}{|l|}{ Type of surgical incision* } \\
\hline Clean (class I) & 3 & 0 & $>0.05$ \\
\hline $\begin{array}{l}\text { Clean-contaminated } \\
\text { (class II) }\end{array}$ & 5 & 1 & \\
\hline Contaminated (class III) & 0 & 0 & \\
\hline Dirty/infected (class IV) & 0 & 0 & \\
\hline $\begin{array}{l}\text { Interventional operation } \\
\text { incisions }\end{array}$ & 5 & 0 & \\
\hline \multicolumn{4}{|l|}{ Operation room } \\
\hline DSA room & 8 & 1 & $>0.05$ \\
\hline Negative pressure room & 4 & 0 & \\
\hline Bedside & 1 & 0 & \\
\hline \multicolumn{4}{|l|}{ Operation time } \\
\hline Less than 1 hour & 5 & 0 & $>0.05$ \\
\hline $1-3$ hours & 5 & 1 & \\
\hline More than 3 hours & 3 & 0 & \\
\hline
\end{tabular}

* incision classification according to the guidelines for surgical site infection prevention and control technology (2010) classification of incision. DSA, digital subtraction angiography.

As the number of COVID-19 patients increased in early January 2020 in Zhejiang, our hospital became a center for COVID-19, especially for the treatment of severe cases within the Zhejiang Province, on account of our multidisciplinary treatment team and the geographic advantage of being distant from the central area and residential districts.

A total of 105 confirmed cases had been admitted to our hospital as of 23 March 2020, of which 75\% were critically ill, most were elderly and presented with comorbidities such as hepatic and renal failure, poor heart function, gastrointestinal bleeding, and malignancies, which made treatment challenging. During hospitalization, a total of 13 temporary or urgent surgeries including emergency cesarean section, emergency intervention hemostasis, emergency endoscopic retrograde cholangiopancreatography (ERCP), temporary pacemaker installation, coronary stent, and emergency colon tumor resection has been performed, as have 2 whole-lung transplantation surgeries to rescue patients with bilateral lung consolidation (Table 1) Two cases were cured and discharged, and the others were discharged after improvement and transferred to further rehabilitation, 30 -day mortality of the emergency surgical is $0 \%$.

We summarized the main process order list of the emergency surgery, clinical departments and surgical nurse to check items by items, including operating rooms and supplies, staff training, transit routes, protective measures, air disinfection, operating room environment etc.; guidelines for perioperative infection Prevention and control measures. IPC department followed up the whole process, verified each patient. the pre-operative and post-operative emergency COVID-19 surgical infection management checklist to ensure successful emergency COVID-19 surgery, a checklist also helps avoid negligence of all aspects of surgical prevention and control as much as possible, not only by having adequate protection measures but also by preventing the spread of coronavirus in all processes to ensure healthcare workers' safety (Tables 2,3).

All procedures performed in this study involving human participants were in accordance with the Declaration of Helsinki (as revised in 2013). The study was approved by ethics board of People's Hospital of Wuhan University (NO.: WDRY2020-K175). Each patient admitted to the hospital with a relevant agreement signed, and the hospital agrees to protect the data privacy.

\section{Discussion}

\section{Emergency preparations for the operating room}

In addition to a negative-pressure operating room being necessary, a digital subtraction angiography (DSA) room should also be prepared. Critically ill patients with COVID-19 may develop multiple organ failure, many elderly inpatients with comorbidities can deteriorate rapidly. Steroid therapy may be needed to relieve cytokine storms, which may cause gastrointestinal bleeding, especially in patients treated with extracorporeal membrane oxygenation (ECMO).

If bleeding cannot be controlled endoscopically, DSA may be required in a state of emergency. Our emergency DSA hemostatic surgery has achieved excellent outcomes, a total of 8 cases successfully underwent operations in DSA room. The DSA interventional therapy has advantages of being safe and reliable, causes little injury or pain, is widely indicated, has significant effects, and no absolute contraindications. 
Table 2 Pre-operation emergency coronavirus disease 2019 (COVID-19) surgical infection management checklist (Zhejiang COVID-19 operation prevention and control experience)

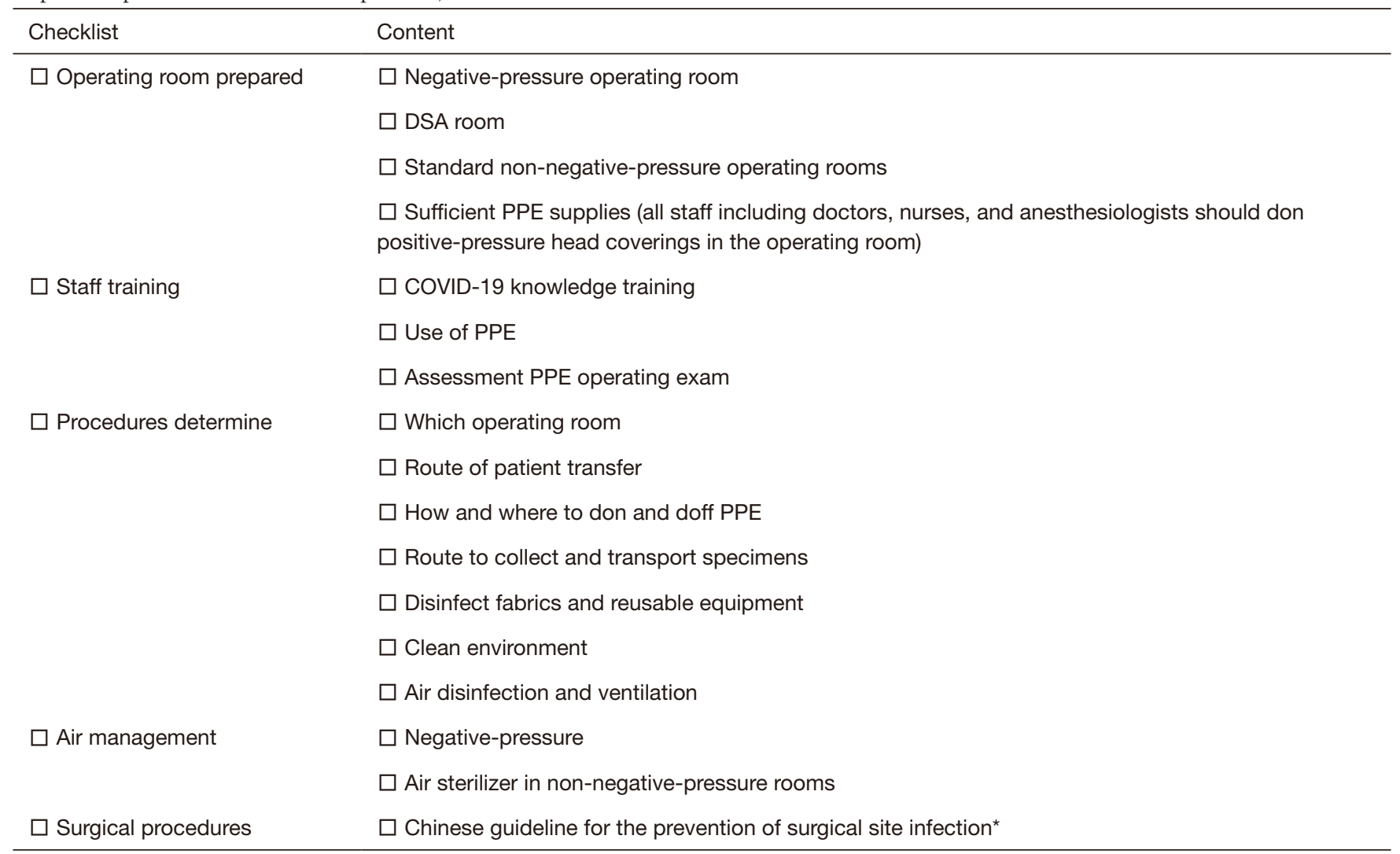

*, all the surgical preoperative and intraoperative procedures follow the Chinese guideline for the prevention of surgical site infection same as the ordinary elective surgery patients. PPE, personal protective equipment.

\section{Staff training and personal protective equipment}

According to the 6th edition of the Coronavirus Pneumonia Prevention and Control Program of China, personal protective equipment (PPE) including disposable caps, N95 medical masks, disposable gowns, gloves, all-inclusive goggles or face masks, and protective clothing should be available for adequate emergency supplies. The main mode of COVID-19 transmission is via respiratory droplets and contact, aerosol transmission is possible with prolonged exposure to high concentrations of aerosols in a relatively closed environment. Other transmission routes need to be clarified (7). During surgery, staff must wear a full set of protective clothing and N95 mask, and a positive-pressure head cover should also be used.

During the viral pneumonia epidemic, the hospital Infection Prevention and Control (IPC) department will train all employees throughout the hospital network via mobile phone educational software, repeated intensive face-to-face and personalized training for various staff, and ensure that every employee is familiar with the guidelines and documents about COVID-19 issued by the government of China. The head of every department is responsible for internally implementing training and transmission of relevant information.

Every staff member who enters the isolation ward needs to be trained in PPE and assessed by the Infection Prevention and Control (IPC) department. Staff are repeatedly shown videos about donning and doffing protective clothing, and emergency surgical personnel are required to dedicate time to improving their protection training.

\section{Developing procedures for emergency surgical infection management}

The IPC department has published multiple documents based on national standards and guidelines, including how 
Table 3 Post-operation emergency coronavirus disease 2019 (COVID-19) surgical infection management checklist(Zhejiang COVID-19 operation prevention and control experience)

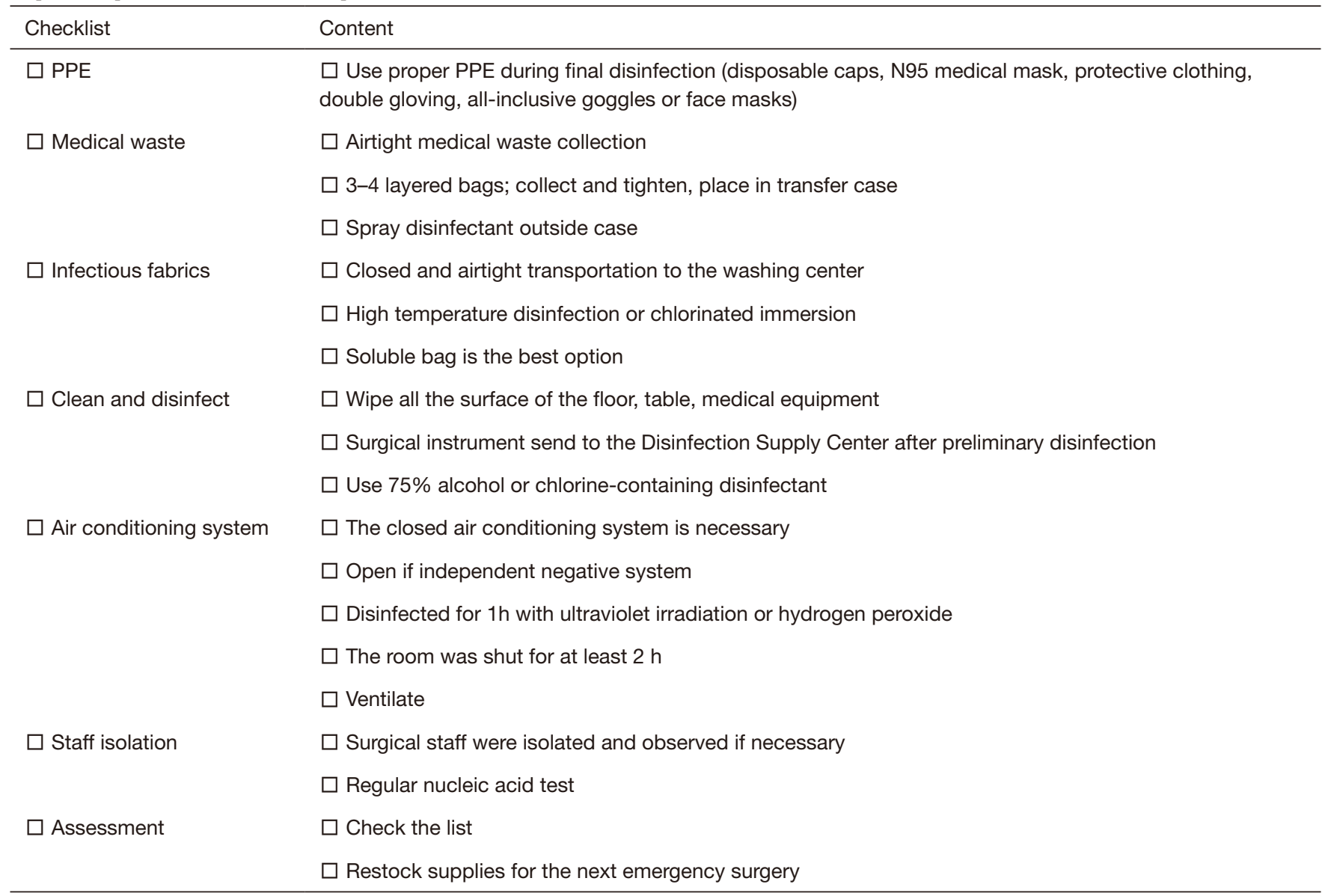

PPE, personal protective equipment.

to don and doff protective equipment, transfer patients for examinations, collect and transfer patient specimens, disinfect fabrics and reusable equipment and goods, keep the environment clean, and so on. All departments are required to strictly adhere to the stipulated procedures.

Surgical specimens are placed individually in doublelayer bags, then in a special specimen transfer box, a "biohazard" label will be fixed to the outside of the box, and then sent to the pathology department after surgery.

All relevant surgery departments will participate in a pre-operative discussion, conduct field inspections, and perform simulation exercises on the routes used for surgical personnel, patients, and goods and equipment. A complete emergency plan is devised through thorough consultation with the specialist. Various conventional standards of pre-, intra-, and post-operative infection prevention and control measures are implemented. For example, according to the
2015 Guidelines for Organ Donation in China, patients with COVID-19 who meet lung transplantation indications through comprehensive evaluation can be immediately treated for lung transplantation, if there is a suitable donor for voluntary organ donation. more than ten hours of operation need to improve each surgical procedures.

\section{Final disinfection and staff isolation}

After surgery, the operating room will undergo thorough disinfection including the medical waste, infectious fabrics, medical equipment, and so on. All surfaces, including floor, operating table, and instrument trays are wiped clean and disinfected. Final disinfection is executed according to the checklist.

Despite surgical staff having adhered to strict protective protocols, a full two 7 days of isolation observation in 
separate building is required. A nucleic acid test must be returned negative in order to allow resumption of general duties after two 7 days.

\section{Surgical site infections (SSIs)}

SSIs occur at the incision site and/or deeper underlying tissue spaces and organs within 30 days of a surgical procedure (or within 90 days for surgeries involving implantation of prosthetic material (8).

SSI is the most common and highest associated health care infection (HAI) in low - and middle-income countries, with an overall incidence of $11.8 \%(1.2-23.6 \%)$. In highincome countries, the incidence of SSI is $1.2-5.2 \%(9,10)$.

The procedure of emergency surgery for patients with COVID-19 follows the requirements of the Chinese guidelines among numerous evidence-based recommendations for prevention of preoperative and intraoperative SSIs $(11,12)$.

Out of 13 patients undergoing emergency surgery for COVID-19, SSi occurred in 1 patient, with an infection rate of $7.6 \%$. The incidence of SSI was significantly higher than that of ordinary patients in the same time in the hospital, which was $0.14 \%$ (12 of 8,314 ordinary elective surgery patients in our hospital). The SSI rate of surgical incision class II in patients with COVID-19 surgery was $20 \%$, which was significantly higher than of elective surgery $(0.15 \%, 6$ of 4,304 ordinary elective surgery patients in our hospital during same time).

Thresholds for surgery during the COVID-19 pandemic should be higher than during normal practice, particularly in men aged 70 years and older. Consideration should be given for postponing non-urgent surgical procedures and promoting non-operative treatment to delay or circumvent the need for surgery (1).

However, the successful implementation of emergency surgery for COVID-19 has had positive significance in reducing the incidence of death in patients with COVID-19 by improving emergency treatment ability in our hospital.

\section{Psychological care and rehabilitation exercise}

The Multi-Discipline Team (MDT) expert team at our hospital has interviewed each patient every day to determine the treatment plan. Anxiety and fear were common in patients with COVID-19 (13). Psychologist dynamic assessed critically ill patients every day and gave timely intervention to prevent the psychological crisis.
Simultaneously, rehabilitation therapists offered bedside rehabilitation assessment and rehabilitation exercise every day to promote functional recovery and patients confidence.

\section{Chinese COVID-19 diagnosis and treatment protocol}

China has continuously updated and revised the COVID-19 Diagnosis and Treatment Protocol, Version 1 to Version 8, and the Prevention and Control Protocol, Version 1 to Version 7 (2), with the understanding of the disease, summarizing previous experience and referring to the guidelines of the World Health Organization and other countries. Adhere to the principle of prevention first, the combination of prevention and treatment, timely detection, rapid disposal, precise management and control, effective treatment, to prevent the import of overseas rebound within China; Emphasis on reducing morbidity and mortality; Emphasis on rehabilitation technology treatment, the role of Chinese medicine. These latest and most effective programs have played an essential role in guiding clinical practice.

The shedding of viral RNA nucleic acid should be combined with antibody serology, aetiology test, iconography, clinical symptoms, comprehensively evaluate the patient's condition, and adjust the treatment and prevention control measures. Two weeks' quarantine for discharged patients was required, and a regular following up was also needed come from the Zhejiang experience.

\section{Summary}

Since 26 January 2020, 30 provinces have initiated level 1 public health responses to control COVID-19, and it is crucial to accelerate the development of vaccines and drugs to defeat COVID-19 (14). It is vital to implement aggressive infection control measures to prevent the spread of COVID-19 via human-to-human transmission (15). Public health authorities need to continuously monitor the developing situation (16).

As a fixed-point hospital that receives and treats COVID-19 patients in the province, all hospital staff play a critical role in infection prevention and control. As of 23 March 2020, the cumulative cure rate of COVID-19 in our hospital is $98 \%$, the patient mortality rate is $0 \%$, and the incidence of COVID-19 infection in staff is $0 \%$.

The DSA interventional surgery is the highest emergency surgery for severe COVID-19 patients, the average operation time is about $1.5 \mathrm{~h}$, and emergency 
surgery for COVID-19 patients in the hospital has been successful and reduced patient deaths. Alongside following the pre-operative and post-operative emergency COVID-19 surgical infection management checklist, IPC staff have conducted a quality inspection and supervision after every emergency surgery, provided necessary feedback, and every emergency surgery has been satisfactory after following the checklist. The emergency surgery is able to performed promptly following surgical planning decisions and the operating room is ready for use at any time. The preparation time for emergency treatment, especially preoperative preparation time has been minimized while the risk of bleeding and hypoxia of patients has also reduced. The Zhejiang COVID-19 operation prevention and control experience checklist has shown feasibility and is effective when accompanied with the surgical preoperative and intraoperative procedures guide.

\section{Acknowledgments}

I wish to express my deepest gratitude to my supervisor, Prof. zuobingchen, who given me the most valuable suggestions and advice, and made necessary corrections. and I also would like to express my great thanks to my whole colleagues, who have generously offered their help with my work.

Funding: The work was supported by a grant from the Zhejiang Key Research and Development Plan Emergency Project (2020C03123-7). And Zhejiang Province Science and technology of traditional Chinese medicine programme (2017ZK1003).

\section{Footnote}

Conflicts of Interest: All authors have completed the ICMJE uniform disclosure form (available at http://dx.doi. org/10.21037/apm-21-430). The authors have no conflicts of interest to declare.

Ethical Statement: The authors are accountable for all aspects of the work in ensuring that questions related to the accuracy or integrity of any part of the work are appropriately investigated and resolved. All procedures performed in this study involving human participants were in accordance with the Declaration of Helsinki (as revised in 2013). The study was approved by ethics board of People's Hospital of Wuhan University (NO.: WDRY2020-K175). Each patient admitted to the hospital with a relevant agreement signed, and the hospital agrees to protect the data privacy.

Open Access Statement: This is an Open Access article distributed in accordance with the Creative Commons Attribution-NonCommercial-NoDerivs 4.0 International License (CC BY-NC-ND 4.0), which permits the noncommercial replication and distribution of the article with the strict proviso that no changes or edits are made and the original work is properly cited (including links to both the formal publication through the relevant DOI and the license). See: https://creativecommons.org/licenses/by-nc-nd/4.0/.

\section{References}

1. COVIDSurg Collaborative. Mortality and pulmonary complications in patients undergoing surgery with perioperative SARS-CoV-2 infection: an international cohort study. Lancet 2020;396:27-38.

2. China NHCo: New coronavirus pneumonia prevention and control program. Availableonline: http://www.nhc.gov. cn/xcs/zhengcwj/202008/0a7bdf12bd4b46e5

3. Riou J, Althaus CL. Pattern of early human-to-human transmission of Wuhan 2019 novel coronavirus (2019$\mathrm{nCoV}$ ), December 2019 to January 2020. Euro Surveill 2020;25:2000058.

4. Backer JA, Klinkenberg D, Wallinga J. Incubation period of 2019 novel coronavirus (2019-nCoV) infections among travellers from Wuhan, China, 20-28 January 2020. Euro Surveill 2020;25:2000062.

5. Hui DS, I Azhar E, Madani TA, et al. The continuing 2019-nCoV epidemic threat of novel coronaviruses to global health - The latest 2019 novel coronavirus outbreak in Wuhan, China. Int J Infect Dis 2020;91:264-6.

6. Wu Z, McGoogan JM. Characteristics of and Important Lessons From the Coronavirus Disease 2019 (COVID-19) Outbreak in China: Summary of a Report of 72314 Cases From the Chinese Center for Disease Control and Prevention. JAMA 2020;323:1239-42.

7. Cowling BJ, Leung GM. Epidemiological research priorities for public health control of the ongoing global novel coronavirus (2019-nCoV) outbreak. Euro Surveill 2020;25:2000110.

8. Borchardt RA, Tzizik D. Update on surgical site infections: The new CDC guidelines. JAAPA 2018;31:52-4.

9. World Health Organization. Report on the endemic burden of health care-associated infection worldwide. 
Geneva: WHO Press, 2011:6-7.

10. Allegranzi B, Bagheri Nejad S, Combescure C, et al. Burden of endemic health-care-associated infection in developing countries: systematic review and meta-analysis. Lancet 2011;377:228-41.

11. Chinese Society of Surgical Infection and Intensive Care, Chinese Society of Surgery, Chinese Medical Association; Chinese College of Gastrointestinal Fistula Surgeons, Chinese College of Surgeons, Chinese Medical Doctor Association. Chinese guideline for the prevention of surgical site infection. Zhonghua Wei Chang Wai Ke Za Zhi 2019;22:301-14.

12. Fields AC, Pradarelli JC, Itani KMF. Preventing Surgical Site Infections: Looking Beyond the Current Guidelines. JAMA 2020; 323:1087-8.

13. Xu K, Cai H, Shen Y, et al. Management of COVID-19:

Cite this article as: Ni L, Zhou Y, Ni Z, Ma Y, Fen H, Wang F, Zhang S, Fei X, Pan Q, Hong X, Qu T, Zhong Z, Chen Z. The emergency surgical infection management checklist of hospitalized patients with coronavirus disease 2019 (COVID-19) in Zhejiang, China. Ann Palliat Med 2021;10(3):3452-3458. doi: 10.21037/apm-21-430 the Zhejiang experience. Zhejiang Da Xue Xue Bao Yi Xue Ban 2020;49:147-57.

14. Deng SQ, Peng HJ. Characteristics of and Public Health Responses to the Coronavirus Disease 2019 Outbreak in China. J Clin Med 2020;9:575.

15. Wu JT, Leung K, Leung GM. Nowcasting and forecasting the potential domestic and international spread of the 2019-nCoV outbreak originating in Wuhan, China: a modelling study. Lancet 2020;395:689-97.

16. Lai CC, Shih TP, Ko WC, et al. Severe acute respiratory syndrome coronavirus 2 (SARS-CoV-2) and coronavirus disease-2019 (COVID-19): The epidemic and the challenges. Int J Antimicrob Agents 2020;55:105924.

(English Language Editor: J. Jones) 